\title{
Simulation numérique des effets visqueux en configuration partiellement cavitante
}

\author{
par T. Maître \& C. Pellone
}

Laboratoire des Ecoulements Géophysiques et Industriels (Institut de Mécanique de Grenoble)

\section{I 口 INTRODUCTION}

\begin{abstract}
La présente étude a pour objectif la modélisation de l'écoulement partiellement cavitant autour de deux profils placés en incidence dans la petite veine du bassin d'essais des carènes (Val de Reuil). Deux codes de calcul sont utilisés, l'un basé sur une méthode d'éléments finis, l'autre sur une méthode intégrale aux frontières (singularités). La modélisation physique consiste en un modèle stationnaire ouvert à interface dans lequel l'écoulement liquide sain extérieur à la cavité et son sillage sont séparés du profil par une ligne de courant issue du point de détachement et se prolongeant jusqu'au bord de fuite sans s'y rattacher. Une analyse critique est proposée sur les problèmes de détachement, de structure interne, de prise en compte du sillage et des pertes, connus pour être des points sensibles de la modélisation.
\end{abstract}

\subsection{Le détachement des cavités}

Le problème de la position du point de détachement est difficile car de faibles variations de la géométrie au voisinage de ce point entraînent des modifications très sensibles sur les formes de cavité. Du point de vue expérimental, on constate que sur des géométries d'inducteur, les bords d'attaque minces entraînent de meilleures capacités d'aspiration en fonctionnement cavitant qu'en fonctionnement subcavitant [1]. Un des points déterminants est la prédiction de la position du point de détachement. Pour des corps à nez hémisphérique, Arakeri [2] propose une corrélation fixant l'écart entre décollement subcavitant et cavitant en fonction du nombre de cavitation et de paramètres ne dépendant que de l'écoulement subcavitant, à savoir: l'écart entre le point de décollement et le point de pression minimum en régime subcavitant et la valeur de la pression au point de décollement subcavitant. La conclusion importante est que le décollement laminaire en écoulement cavitant est indépendant du nombre de Reynolds et se décale vers l'amont lorsque le nombre de cavitation diminue ; l'écart entre le détachement de la cavité et le décollement laminaire est indépendant du nombre de cavitation et diminue quand le nombre de Reynolds augmente. Des expériences menées par Franc et Michel [3] confirment ces dépendances en nombre de cavitation et de Reynolds pour un cylindre.

D'un point de vue numérique, la position du point de détachement a une influence importante sur la qualité des résultats. Un certain nombre de critères ont été proposés pour prédire le détachement des cavités dans les modélisations :

- Les critères de Villat [4] et de Pellone [5] : au point de détachement, le premier consiste à assurer la continuité de la courbure, le second celle des pressions; ces deux critères sont équivalents et donnent des résultats médiocres.

- Le critère du coefficient de pression minimum : il impose le point de détachement de la cavité au point de pression minimum en régime subcavitant. Ce critère est plus ou moins satisfaisant suivant les géométries.

- Le critère du départ tangent : il consiste à chercher le point pour lequel la première facette de cavité est tangente à la facette précédente sur le corps. Ce critère semble assez sensible à la discrétisation.

- Le critère de la couche limite : il consiste à coupler un calcul de couche limite et un calcul cavitant pour déterminer itérativement le point de décollement laminaire. Ce critère est le seul s'appuyant sur l'expérience.

\subsection{Prise en compte du sillage et des pertes}

Les formes de cavitation sont extrêmement variables suivant les configurations d'écoulement. Les paramètres qui jouent un rôle important sont le taux de germes, le nombre de cavitation, l'incidence (pour un profil), le nombre de Reynolds, 
la géométrie du corps étudié [3]. En régime de cavitation partielle, il apparaît deux structures de poche bien distinctes : soit une région purement vapeur, soit une région diphasique pour des incidences plus fortes. Il semble possible que ces deux cas correspondent respectivement à la désignation «non burst " et «burst " utilisée par Kawamani et Kato [6]. Ces auteurs montrent que les caractéristiques d'un écoulement cavitant dépendent en grande partie du type de couche limite développée dans l'écoulement subcavitant. Dans le cas d'une couche limite laminaire générant une région importante de recirculation, la cavité est relativement opaque et les auteurs parlent d'une cavitation par amas de bulles (" cluster bubble cavitation »).

Les modélisations de type interface font le plus souvent l'hypothèse de l'écoulement potentiel. Les méthodes proposées sont alors les éléments finis ou les équations intégrales aux frontières. On distingue essentiellement les modèles fermés et ouverts, les codes prédictifs et non prédictifs. D'une façon générale, les modèles fermés sont prédictifs mais peu réalistes et les modèles ouverts sont faiblement ou complètement prédictifs mais plus proches des résultats expérimentaux. Les principaux modèles trouvés dans la littérature sont les suivants :

- Modèle fermé sans sillage. Dans ce modèle aucun sillage de cavité n'est présent.

- Modèle fermé à sillage proche [7]. Une condition à la limite particulière permet de passer régulièrement de la condition de cavité à la condition de glissement sur la surface mouillée du profil à l'aval ; elle permet de représenter la recompression de l'écoulement moyen dans le sillage proche de la cavité. Un degré de liberté est introduit au moyen de la longueur de ce sillage proche qui reste réglable. A longueur de poche donnée, les vitesses sont surestimées sur la cavité et conduisent à des nombres de cavitation trop élevés, surtout lorsque les longueurs de poche sont importantes.

- Modèle ouvert à sillage lointain constant calculé [8]. On impose un détachement tangent de la cavité en un point déterminé par le critère du décollement laminaire. Ce code prédictif donne de bons résultats.

- Modèle sans sillage à ouverture imposée. Ce type de modèle permet de simuler un déficit de vitesse en aval de la cavité sans pour autant utiliser de sillage. L'ouverture à l'aval de la cavité est prise proportionnelle à la longueur de cavité et au carré de l'incidence d'après des mesures sur profil fin. Ce modèle est faiblement prédictif.
- Modèle ouvert à cavité et sillage imposés par la pression. En partant de pressions expérimentales obtenues sur la cavité et son sillage, la géométrie de la ligne de courant simulant ces deux régions est déterminée. Juste en aval de la cavité cette ligne se rapproche rapidement du profil dans une région où les pressions imposées augmentent rapidement. C'est la région du sillage proche, où la vapeur se condense et l'épaisseur de déplacement associée diminue. Au delà, cette ligne s'éloigne lentement du profil. C'est la région du sillage lointain où la couche limite turbulente se développe et l'épaisseur de déplacement associée augmente. Ce modèle non prédictif conduit à des vitesses, rentrant dans le profil au voisinage du sillage proche et sortant du profil au voisinage du sillage lointain, cohérentes avec l'évolution des épaisseurs de déplacement décrite précédemment.

- Modèle fermé à sillages proche et lointain imposés par une variation d'épaisseur de nappe [9]. Ce modèle introduit une notion d'épaisseur de la nappe de courant tout à fait analogue aux épaisseurs des tubes de courant introduites dans les modélisations quasi tridimensionnelles d'écoulements dans les turbomachines. La variation d'épaisseur, en modifiant le champ de vitesse par conservation du débit dans la nappe, permet de représenter l'effet du déficit de vitesse dans le sillage de la cavité et sur les parois de la veine. Deux coefficients permettent de caler le sillage proche et lointain par rapport à l'expérience; le modèle est donc faiblement prédictif.

Les deux modèles utilisés dans la présente étude sont ouverts à sillages proche et lointain calculés. Ils sont faiblement prédictifs car une relation empirique permet de déduire l'épaisseur de déplacement dans le sillage en écoulement cavitant en fonction de son équivalent en écoulement subcavitant.

\section{II — FORMULATION DU PROBLÈME ET MODÉLISATION}

\subsection{Formulation}

La veine d'essais est une veine hydrodynamique fermée de hauteur $H=480 \mathrm{~mm}$ et de largeur totale $600 \mathrm{~mm}$ (fig. 1). Pour rendre l'écoulement bidimensionnel dans la zone d'essais, une plaque plane verticale est disposée dans le sens de l'écoulement de façon à partager la veine en deux sous-

1. Configuration de la veine d'essais.

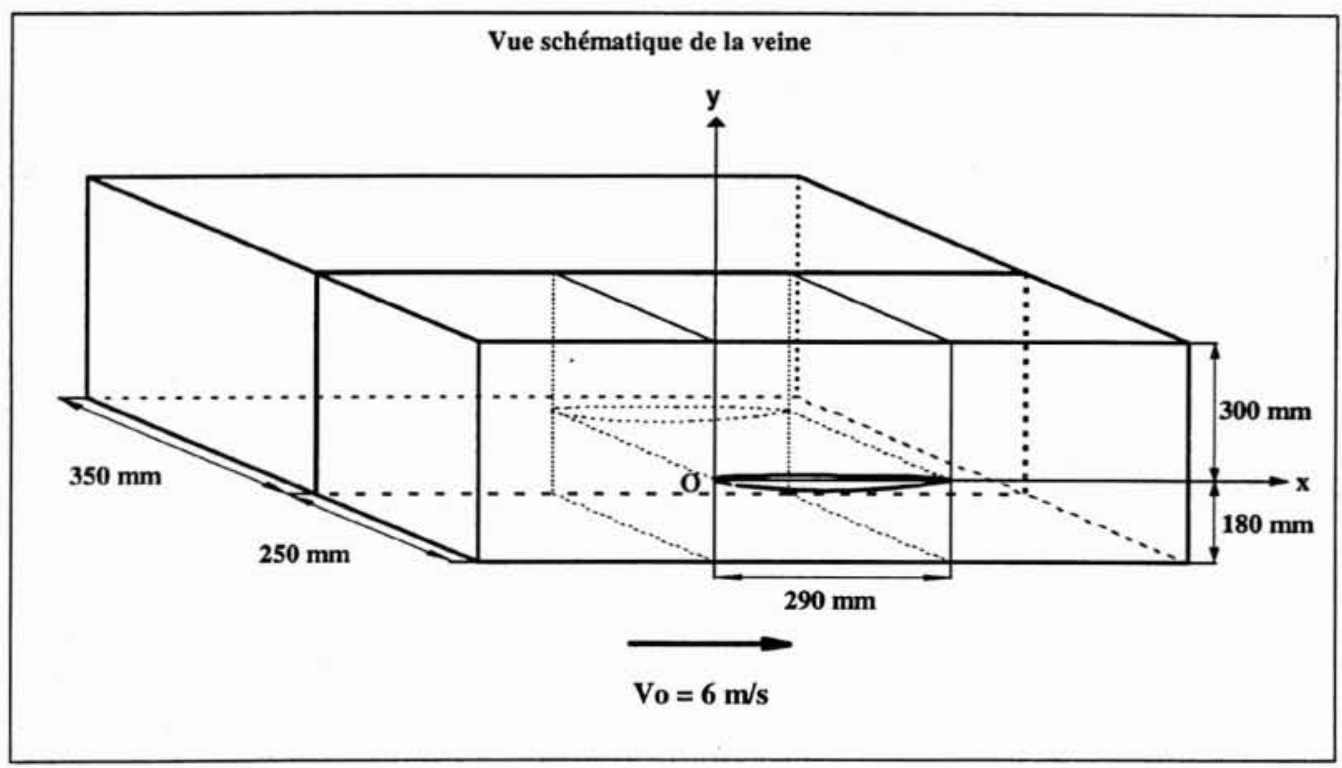


veines : une première sous-veine, de largeur $D=250 \mathrm{~mm}$, contient une aile rectangulaire dont le profil bidimensionnel est uniforme selon l'envergure ; une deuxième sous-veine, large de $350 \mathrm{~mm}$, laisse libre le passage de l'eau. L'axe de rotation de l'aile est repéré par le point milieu de la corde de longueur $c=290 \mathrm{~mm}$ d'un profil bidimensionnel ; ce point est situé à une profondeur d'immersion $h=300 \mathrm{~mm}$ et sert de centre de rotation pour les mises en incidence $\alpha$ de l'aile. L'écoulement, loin en amont, de vitesse $V o=6 \mathrm{~m} / \mathrm{s}$, est obtenu unidirectionnel à l'aide d'un convergent dont la sortie est située à la distance $X o=2,25 \mathrm{~m}$ de l'axe de rotation de l'aile. Dans une section quelconque de l'aile, on définit un repère orthonormé direct $O x y$, ayant pour origine le point $O$ bord d'attaque du profil, dont l'axe $O x$ est dirigé suivant le vecteur vitesse amont $\overrightarrow{\mathbf{V}}_{\mathrm{o}}$ et l'axe $\mathrm{Oy}$ ascendant vertical ; l'angle d'incidence $\alpha$ du profil bidimensionnel est compté positivement dans le sens des aiguilles d'une montre. On désigne par $x$ et $y$ les coordonnées d'un point $M$ du plan bidimensionnel ainsi défini. Les essais sont effectués à la température ambiante de $20^{\circ} \mathrm{C}$, ce qui donne pour l'eau, une viscosité cinématique $v=1,00510^{-6} \mathrm{~m}^{2} / \mathrm{s}$. Dans le voisinage de l'aile, ce type de configuration permet d'avoir des épaisseurs de couche limite pratiquement constantes sur le fond et le plafond ; pour les deux profils étudiés, les mesures expérimentales ont donné pour ces épaisseurs une valeur commune de $\delta=35 \mathrm{~mm}$. Sur les parois latérales de la veine et sur la plaque plane, les conditions expérimentales autorisent la non prise en compte des épaisseurs de couche limite. En effet, le bord d'attaque de la plaque plane n'étant situé qu'à environ une corde en amont du bord d'attaque du profil, l'épaisseur de couche limite développée sur celle-ci au voisinage de l'aile, est relativement très petite et de fait largement négligeable. Sur la paroi latérale extérieure, un dispositif de soufflage de la couche limite par injection d'eau permet, juste un peu en amont de l'aile, de réduire assez fortement son épaisseur pour pouvoir aussi la négliger. Dans le développement de l'étude, seules les couches limites développées sur le fond et le plafond, ainsi que celles développées sur le profil seront donc prises en compte.

Deux profils de types différents ont été étudiés. Le premier, nommé profil A, a une épaisseur maximale de $11 \%$ et présente une cambrure négative (fig. 6). Ce profil est le résultat d'un calcul potentiel bidimensionnel inverse qui fournit la cambrure et l'épaisseur à partir d'une répartition de pression imposée. Cette répartition est caractéristique de celle obtenue pour la section de pied de pale d'une hélice marine. L'intérêt d'un tel profil est qu'il possède une répartition de charge uniformément distribuée ; sa cambrure inverse lui confère une structure très différente de celles utilisées habituellement. Il est donc très différent du deuxième profil classique, nommé profil $C$. Ce dernier a une épaisseur maximale de $6 \%$ et fait partie de la famille NACA66 modifiée (fig. 5).

On suppose qu'une cavité prend naissance au point de détachement $\omega$ (fig. 2), se développe à l'extrados du profil jusqu'au point $C$ et se rattache sur la ligne de sillage $S F$ (ligne de courant dans le cas stationnaire), par l'intermédiaire d'une zone de raccord $C S$. Cette zone n'est introduite que pour régulariser les conditions aux limites de nature différente sur la cavité (pression constante) et le sillage (ligne de courant). La loi d'ouverture sur le sillage à l'aval de la zone de raccord est obtenue par le calcul de couche limite présenté au $\$ 3.1$ et permet ainsi de prendre en compte les effets visqueux par l'intermédiaire de la surface de déplacement calculée. Le calcul est alors réalisé dans les conditions de l'écoulement potentiel, mais le contour réel des frontières est remplacé par un contour fictif épaissi de l'épaisseur de déplacement. La présente étude est faite en considérant la pression relative dans la cavité comme une inconnue du problème, la longueur de cavité étant alors imposée. La longueur de cavité ramenée à la corde du profil est donnée par
$L_{c}=X_{c}-X_{w}$; on désignera par $L_{s}=X_{s}-X_{w}$ la longueur étendue de cavité. Le coefficient de pression étant défini par $C_{p}=2\left(p-p_{0}\right) /\left(\rho V^{2}\right)$, la pression relative dans la cavité est caractérisée par le nombre de cavitation $\sigma=2\left(p_{0}-p_{c} /\left(\rho V^{2}\right)\right.$, $p_{c}$ étant la pression dans la cavité. Pour le cas de l'écoulement potentiel stationnaire (fluide parfait, écoulement irrotationnel), on désigne par $\phi$, le potentiel des vitesses qu'on peut écrire sous la forme $\phi=\phi+V o x, \phi$ désignant le potentiel de perturbation. Dans ces conditions $\phi$ satisfait l'équation de Laplace dans tout le domaine fluide. Le champ de vitesse est alors donné par $\vec{V}=\vec{V} 0+\vec{v}$, avec $\overrightarrow{\mathbf{v}}=\overrightarrow{\Delta \phi}$. Les conditions aux limites sont alors données par les relations suivantes :

Une condition de glissement sur la partie mouillée du profil $\left(F_{0} A \omega\right)$ :

$$
\partial \phi / \partial n=-\vec{V} \mathbf{o} \cdot \vec{n}
$$

Une condition de glissement, plus une condition de pression constante sur la cavité $(\omega C)$ :

$$
\begin{gathered}
\partial \phi / \partial \mathbf{n}=-\overrightarrow{V_{o}} \cdot \overrightarrow{\mathbf{n}} \\
\partial \phi / \partial \mathrm{t}=-V_{0} \sqrt{1+\sigma}-\overrightarrow{V_{0}} \cdot \overrightarrow{\mathbf{t}}
\end{gathered}
$$

Une loi de comportement de la vitesse normale sur la zone de raccord $(C S)$ :

$$
\partial \Phi / \partial n=\vec{v} \cdot \vec{n}=[1-f(u)] \vec{v}_{c} \cdot \vec{n}_{c}+f(u) \vec{v}_{s} \cdot \vec{n}_{s}
$$

Une condition de glissement sur le sillage $(S F)$ :

$$
\partial \phi / \partial \mathbf{n}=-\overrightarrow{V_{0}} \cdot \overrightarrow{\mathbf{n}}
$$

La relation (3) est l'écriture sur une ligne de courant (cas stationnaire) de la relation $C_{p}=-\sigma$. Dans la relation (4) l'indice $C$ indique que les grandeurs sont prises au point $C$ de fin de cavité et l'indice $S$ au point $S$ de début de sillage; $u=\left(s-s_{c}\right) /\left(s_{s}-s_{c}\right)$ est l'abscisse curviligne adimensionnelle appartenant à 1 'intervalle $[0,1]$. La fonction $f$ est introduite uniquement pour régulariser le passage d'une condition de pression constante sur la cavité à une condition de glissement sur le profil. Cette fonction est $a$ priori arbitraire mais doit être continue et avoir pour valeurs aux bornes de l'intervalle : $f(0)=0, f(1)=1$. Ainsi, partout sur la zone de raccord, la vitesse normale s'exprime uniquement en fonction des vitesses normales au point $C$ et au point $S$. Au point $S$ la relation (2) impose $\vec{V} \vec{n}_{s}=0$. En conséquence, puisque $V_{c} n_{c} \rightarrow 0$ lors de la convergence de la procédure itérative de déformation de la cavité (cf $\S 3.2$ ), la relation (4) assure ainsi en fin de convergence le glissement du fluide partout sur la zone de raccord. Cette convergence est d'autant plus régulière que $f$ est régulière. Dans cette étude $f$ est prise linéaire $(f(u)=u$ ). Deux autres conditions sont à ajouter, l'une traduisant le glissement du fluide sur les parois de la veine, l'autre la continuité de pression au bord de fuite du profil (condition de Joukovski). La première est réalisée de façon spécifique pour les deux méthodes utilisées ( $\mathrm{cf}$ § 2.2), la deuxième est satisfaite de façon itérative à l'aide de la procédure de déformation de la cavité.

\subsection{Méthode intégrale et méthode des éléments finis}

Le problème posé au $\S 2.1$ se réduit essentiellement à chercher le potentiel $\phi$ satisfaisant l'équation de Laplace partout dans le domaine fluide, ainsi que les conditions aux limites (1) à (5) et les deux conditions supplémentaires. La méthode des singularités (équations intégrales du problème) utilise une double répartition de singularités de type simple couche et double couche réparties sur les frontières. La détermination de ces distributions permet d'accéder aux champs du potentiel et de vitesse partout dans le domaine fluide. La méthode des images permet de prendre en compte automatiquement le fond et le plafond de la veine. Les méthodes 
classiques de discrétisation et de résolution avec minimisation des erreurs de discrétisation sont utilisées [7].

La méthode des éléments finis nécessite un maillage du domaine bidimensionnel. Les équations du problème aux limites sont résolues à l'aide de la fonction de courant associée. Pour cela une formulation variationelle est utilisée : elle associe au problème une fonctionnelle dont la minimisation conduit à la solution. Une discrétisation, du point de vue mathématique, permet de résoudre le problème dans un sous-espace vectoriel de dimension finie, inclus dans l'espace vectoriel des solutions.

\section{III —DESCRIPTION DU MODÈLE}

\subsection{Prise en compte des couches limites}

En ce qui concerne les couches limites développées sur le fond et le plafond de la veine, une étude préliminaire des différentes possibilités de formulation a été faite. Ces couches limites, se développant loin en amont à la sortie du convergent, donnent un rétrécissement fluide pratiquement constant dans la zone de l'aile. Dans ces conditions, l'évaluation de ce rétrécissement peut se faire en utilisant les résultats de couche limite turbulente obtenus pour une plaque plane lisse. Avec les valeurs indiquées au $\$ 2.1$, la distance de la fin du convergent au bord d'attaque du profil est de $2,105 \mathrm{~m}$ et celle au bord de fuite de $2,395 \mathrm{~m}$, ce qui donne des nombres de Reynolds respectifs de $1,2610^{7}$ et $1,4310^{7}$. Pour la valeur de $\delta=35 \mathrm{~mm}$, la valeur moyenne, obtenue par différentes formules, pour l'épaisseur de déplacement $\delta^{*}$ est de $4 \mathrm{~mm}$. Sa prise en compte dans le calcul potentiel se fera simplement de la façon suivante : la hauteur de la veine $(480 \mathrm{~mm})$, sera rétrécie de $4 \mathrm{~mm}$ au plafond et de $4 \mathrm{~mm}$ au fond. L'écoulement potentiel est simulé ainsi dans une veine équivalente de $472 \mathrm{~mm}$ de hauteur. Avec ces considérations, la hauteur adimensionnelle de la veine équivalente est donnée par $H_{0}=\left(H-2 \delta^{*}\right) / c$.

En ce qui concerne le profil, un calcul de couche limite est nécessaire pour prédire le point de détachement des cavités partielles et aussi pour servir de base à la prise en compte des sillages ouverts en aval des cavités. Dans le premier cas, un calcul laminaire avec prédiction du point de décollement suffit ; dans le deuxième cas, on utilise la prédiction de la couche limite turbulente d'extrados en régime subcavitant pour construire la cavité à sillage ouvert (zone $S F$ sur la figure 2). Que ce soit dans l'un ou l'autre cas les méthodologies utilisées font appel à des procédures itératives de résolution entre le calcul de la couche limite et le calcul de l'écoulement sain cavitant. Le programme de couche limite utilisé est celui développé dans l'étude [9]. Il permet la modélisation des couches limites en écoulement bidimensionnel ou axisymétrique en fluide incompressible, en régime laminaire ou turbulent. Pour les deux profils étudiés, la couche limite intrados étant bien plus mince qu'à l'extrados, est négligée. A l'extrados, on suppose que la couche limite présente un bulbe de décollement laminaire, qui transite et recolle rapidement sur le profil [6]. Pour obtenir la couche limite turbulente après le recollement, on utilise une méthode approchée qui consiste à faire démarrer le calcul juste après le décollement laminaire déterminé précédemment.

\section{- 3.2 Modèle ouvert avec prise en compte des pertes générées par la cavité}

Un calcul préliminaire de couche limite en configuration subcavitante est effectué ; en plus de la position du point de décollement laminaire de la couche limite, ce calcul permet

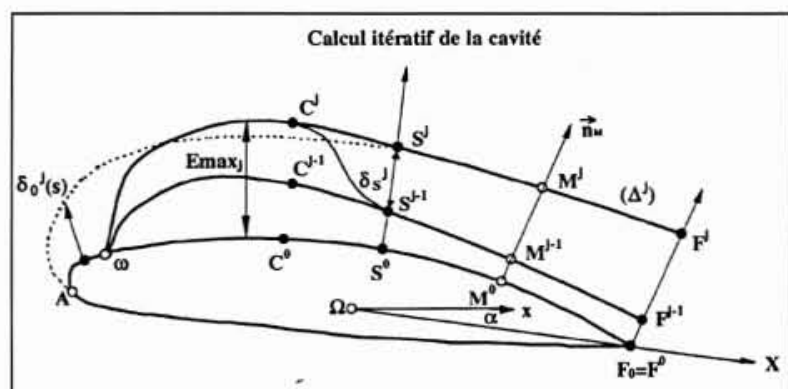

2. Schéma itératif des géométries de cavité.

d'avoir l'épaisseur de déplacement $\delta_{0}(\mathbf{s})$ partout sur le profil. Le point de détachement, étant fixé soit par le critère du départ tangent, soit par celui de couche limite (décrits au $\S 1.1$ ), le calcul géométrique de la cavité, de la zone de raccord et du sillage se fait par une procédure itérative décrite ci-après. Les géométries étant connues à l'itération $j-1$, les conditions aux limites du $\S 2.1$ sont appliquées, sauf en ce qui concerne le glissement du fluide sur la cavité en propre (2). A l'itération courante $\mathrm{j}$, afin que cette condition (glissement du fluide sur la cavité et la zone de raccord) soit satisfaite, la géométrie de la cavité et de la zone de raccord est déformée, ce qui procure les nouvelles géométries de cavité $\omega C^{j}$ et de raccord $C^{j} S^{j}$ (fig. 2). A l'aval du point $S^{j}$ la courbe de sillage $\left(\Delta^{j}\right)=\left(S^{j} F^{j}\right)$ est simplement prise confondue avec la partie correspondante de la courbe obtenue par épaississement du profil à l'aide de la loi d'épaisseur $\delta_{j}^{j}(s)$ mesurée suivant la normale au profil. La loi d'épaisseur $\delta(s)$ à l'itération $j$ est déduite de la loi subcavitante $\delta_{0}(s)$ de la façon suivante. On suppose que l'interaction de la couche limite avec la présence de la cavité peut être prise en compte par modification de la loi subcavitante $\delta_{0}(s)$. L'idée est de dire que la loi d'épaisseur dans le cas cavitant dépend de la loi subcavitante et de l'épaisseur maximum de la cavité Emax mesurée suivant la normale au profil. Pour le type de profils étudiés, les résultats expérimentaux [6] montrent que la loi cavitante peut être déduite de la loi subcavitante simplement en multipliant cette dernière par un coefficient qui varie linéairement en fonction de $E$ max. Ainsi à chaque itération $j$ la loi cavitante s'écrit

$$
\delta_{0}^{j}(s)=\left[a+b \cdot E \max _{j}\right] \cdot \delta_{0}(s)
$$

Les résultats expérimentaux fournissent simplement par extrapolation les valeurs des deux paramètres $a$ et $b: a=1$ et $b=40$. Au départ, à l'itération (0), la cavité, la zone de raccord et le sillage sont confondus avec le profil et forment la ligne initiale $\left(\omega C^{0} S^{0} F^{0}\right)$; à la première itération, le problème est résolu et la cavité est déformée de façon à ce qu'elle se referme sur le profil au point $S^{0}$; ainsi une première évaluation de l'épaisseur maximum de cavité Emax est obtenue, ce qui permet, à l'aide de la relation (6), d'initialiser à l'itération (1) le calcul de la cavité en modèle ouvert. Aux itérations courantes $[j \geq 1]$, la ligne $\left(\omega C^{-1} S^{j-1} F^{j-1}\right)$ étant supposée connue à l'itération $(j-1)$, la procédure se développe en trois étapes.

1) La cavité est déformée de façon à ce qu'elle se referme par la zone de raccord au point $S^{j-1}$; on obtient alors le contour intermédiaire $\left(\omega C S^{j-1} F^{j-1}\right)$.

2) L'épaisseur maximum de la cavité à l'itération $(j)$ en est déduite ; en utilisant la relation (6) la courbe $\left(\Delta^{j}\right)=\left(S^{j} F^{j}\right)$ est ainsi déterminée.

3) La zone de raccord $C^{j} S^{j}$ à l'itération $(j)$, est alors construite à partir de la courbe $C S^{j-1}$, simplement en rattrapant le jeu $\delta_{\mathrm{s}}^{j}=S^{j-1} S^{j}$ à l'arrière de la zone de raccord, linéairement suivant l'abscisse curviligne et de manière à respecter la position du point $C$. 


\section{IV — RÉSULTATS}

Les figures 3 et 4 présentent respectivement les lois $L(\sigma)$ et $C z(\sigma)$ pour le profil $C$ placé à $4^{\circ}$ d'incidence. Sur chaque figure, les quatre courbes en traits pleins correspondent aux deux méthodes et aux deux critères de détachement. Les points correspondent soit à des longueurs de cavité imposées $[0,1 ; 0,2 ; 0,3 ; 0,4 ; 0,5 ; 0,6]$, soit à des valeurs du nombre de cavitation imposées $[1,31 ; 1,09 ; 0,98 ; 0,89$ : points fléchés]. Sur la courbe 4 , les valeurs expérimentales de portance sont indiquées par un faisceau de trois courbes pointillées représentant la valeur moyenne et l'écart type de la mesure.

On constate sur les deux figures que les résultats obtenus pour chaque méthode ne dépendent pas du critère utilisé pour déterminer la position du point de détachement. En effet les deux critères donnent des points de détachement très voisins et très peu dépendants des longueurs de cavité.

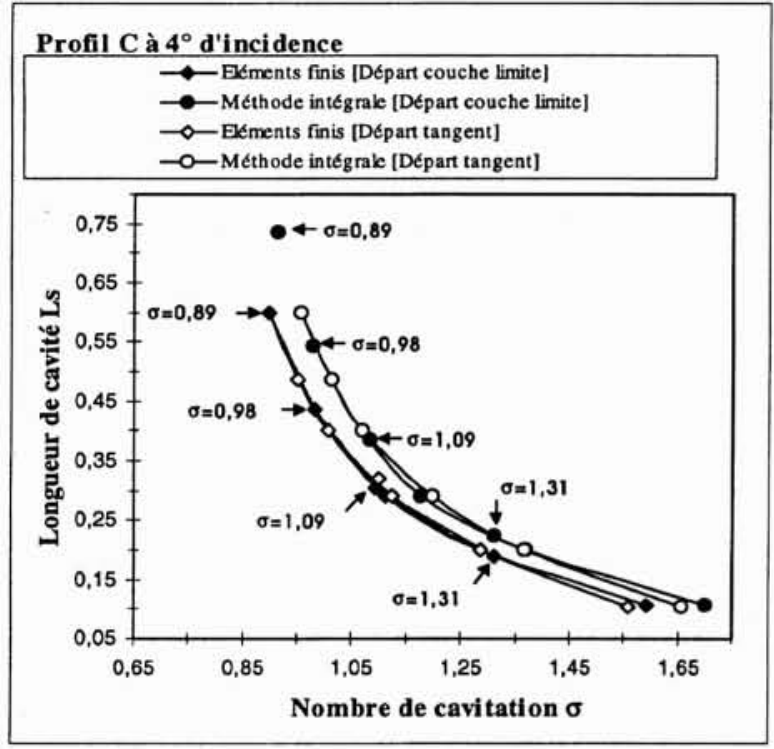

3. Courbes $L(\sigma)$ pour le profil $C$.

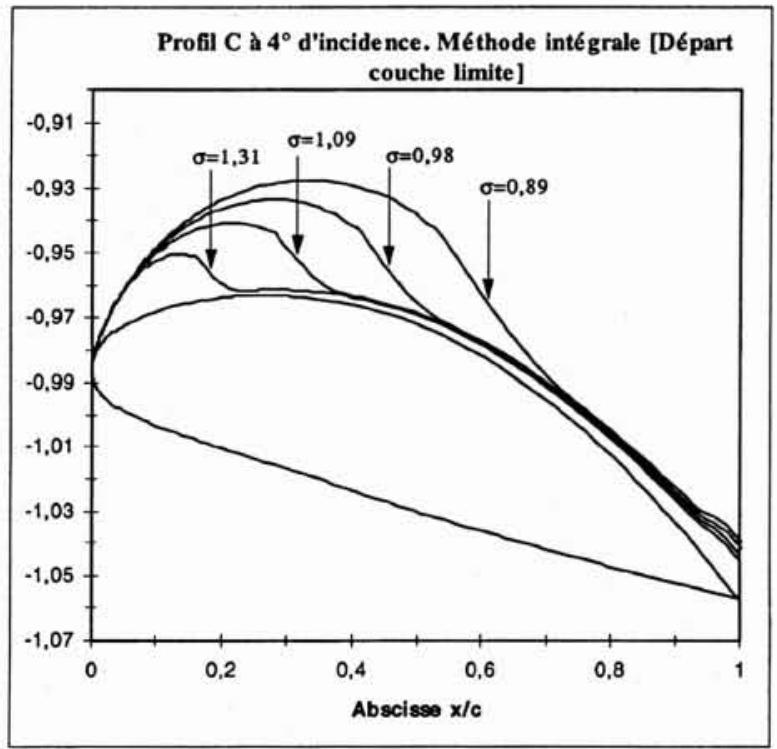

5. Forme des poches pour le profil $C$.
Ce point de détachement est très voisin du point de décollement laminaire subcavitant. La méthode intégrale conduit à des poches plus longues que celle des éléments finis et à des coefficients de portances plus élevés. Les coefficients de portance donnés par les deux méthodes augmentent sensiblement lorsque les poches deviennent plus longues. Globalement, les coefficients de portance fournis par la méthode intégrale sont plus proches des valeurs expérimentales, présentant un écart maximum dont la valeur moyenne relative est de $5 \%$. La figure 5 présente les poches obtenues sur le profil $C$ avec la méthode intégrale alliée au critère de couche limite pour quatre valeurs du nombre de cavitation. Au nombre de cavitation $\sigma=0,89$ l'épaisseur de déplacement vaut $2 \%$ de la corde au bord de fuite (pour mémoire, on trouve expérimentalement dans l'étude [6] une épaisseur de $2 \%$ pour $\sigma=0,87$ ). La figure 6 présente les poches obtenues sur le profil $A$ avec les deux méthodes alliées au critère de couche limite pour quatre valeurs du nombre de cavita-

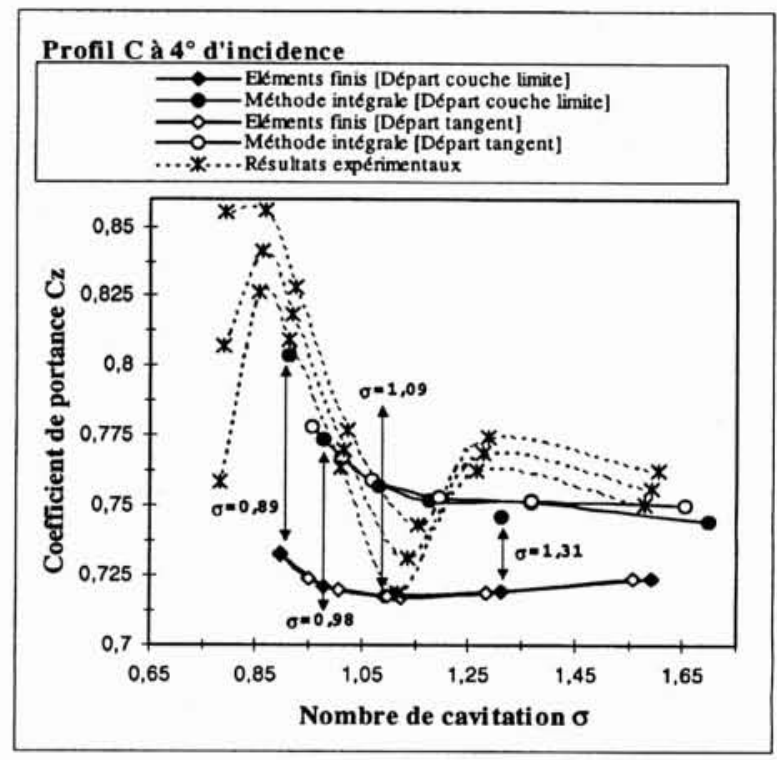

4. Courbes $C z(\sigma)$ pour le profil $C$.

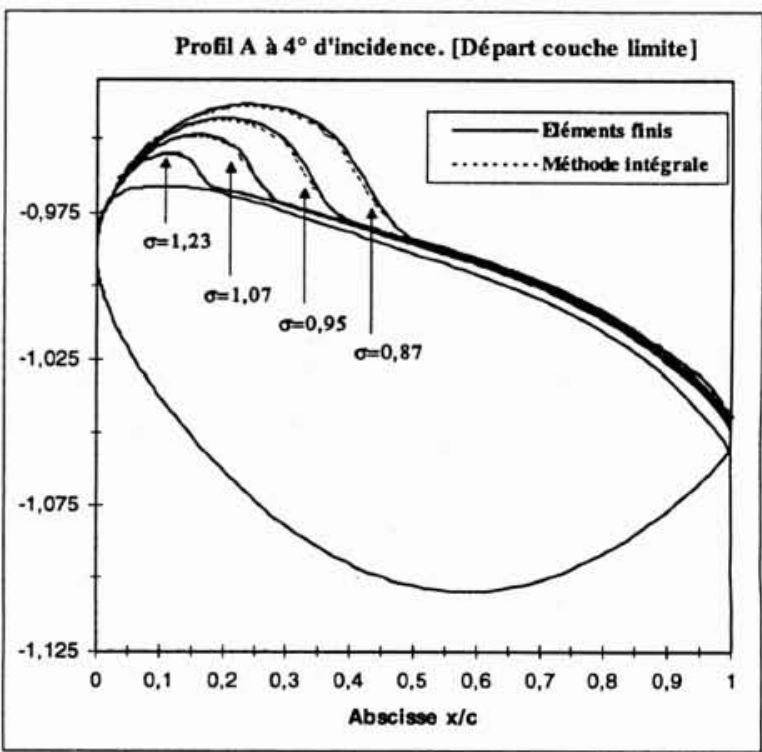

6. Forme des poches pour le profil $A$. 


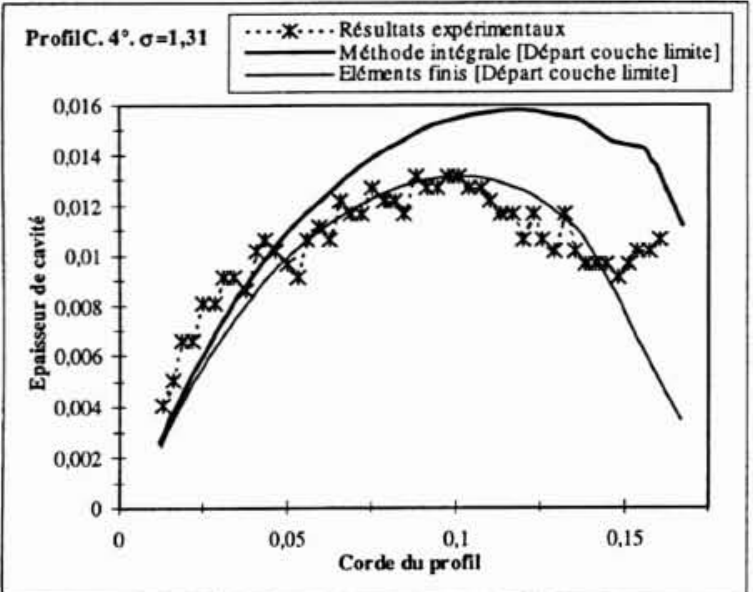

7. Comparaison des formes de poches.

tion $[1,23 ; 1,07 ; 0,95 ; 0,87]$. Les poches obtenues par les deux méthodes sont très voisines, particulièrement pour $\sigma>1$.

La figure 7 présente une comparaison des poches expérimentales et calculées sur le profil $C$ dans le cas de deux valeurs du nombre de cavitation. $A \sigma=1,31$ (poche de $20 \%$ de corde), la poche obtenue par la méthode des éléments finis correspond bien avec la poche expérimentale sauf au voisinage du bord d'attaque où c'est la méthode intégrale qui donne le détachement le plus réaliste. $A \sigma=0,89$, la méthode intégrale donne les meilleurs résultats avec une poche un peu plus fine que la poche expérimentale $(3,5 \%$ contre $4 \%$ ). Notons qu'une épaisseur de déplacement un peu plus faible au bord de fuite conduirait à une poche plus épaisse, améliorant ainsi le résultat.

\section{V - CONCLUSION}

Une modélisation stationnaire d'une cavité représentée par une interface de glissement est proposée dans cette étude. Deux méthodes numériques, différentes par leur fondement mathématique et par les techniques de discrétisation utilisées, sont mises en œuvre sur les mêmes cas de calcul. Une modélisation originale du sillage proche et lointain a été élaborée, sur la base de mesures expérimentales extraites de la littérature. Cette approche, faiblement prédictive, tente de reproduire globalement l'effet du sillage en "épaississant » le profil des épaisseurs de déplacement en fonction de la taille de la cavité. Le point de détachement est obtenu par le critère du décollement laminaire ou celui du départ tangent. L'écart maximum de $5 \%$ sur les coefficients de portance est remarquable. Les poches longues sont légèrement sous-estimées, ce qui laisse entendre que la couche limite en aval de la cavité est un peu surestimée.

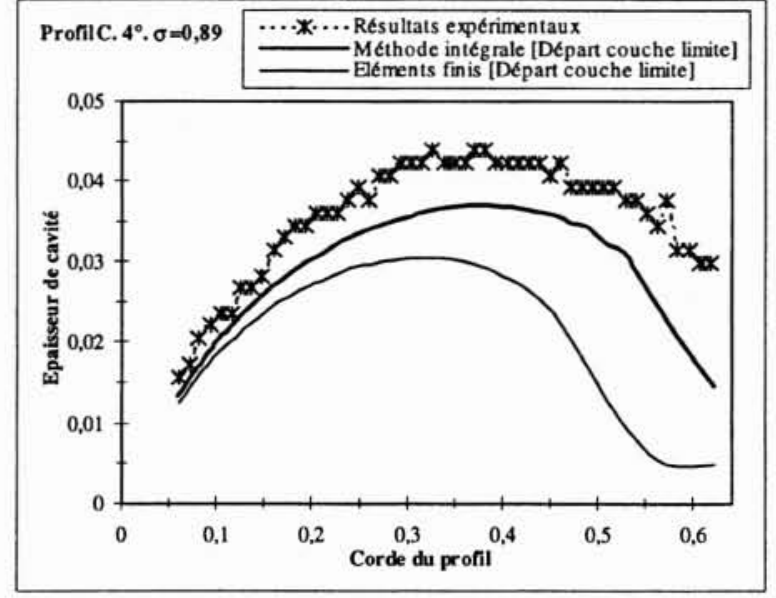

\section{BIBLIOGRAPHIE}

[1] Catelan F.-X. (1996). - Modélisation bidimensionnelle de la cavitation partielle dans les turbomachines. Thèse de doctorat, Institut National Polytechnique de Grenoble, Grenoble, mai 1996.

[2] ARAKERI V.-H., AcostA A.-J. (1976). - Cavitation inception observations on axisymmetric bodies at supercritical Reynolds number. Journal of Ship Research, Vol. 20, $\mathrm{N}^{\circ} 1$, pp. 40-50.

[3] Franc J.-P., Michel J.-M. (1985). - Attached cavitation and the boundary layer : experimental investigation and numerical treatment. Journal of Fluid Mechanics, Vol. 154, pp 63-90.

[4] Villat H. (1914). - Sur la validité des solutions de certains problèmes d'hydrodynamique. Journal de Mathématiques Pures et Appliquées, Vol. 6, p. 10, 1914.

[5] Pellone C. (1981). - Application de la méthode des singularités au calcul des structures supercavitantes en théorie nonlinéaire. Thèse de docteur ingénieur de l'UJF et l'INPG, Grenoble, juin 1981.

[6] Kawamani Y., Kato H., Yamaguch H., Maeda (1995). An experimental investigation of flow field around sheet cavity on foil section. Department of naval architecture and ocean engineering, The university of Tokyo, Hongo, Bunkyo-Ku, Tokyo 113, Séminaire LEGI, octobre 1995.

[7] Pellone C., Rowe A. (1988). - Effect of Separation on Partial Cavitation. Journal of Fluids Engineering, Vol. 110, pp. 182-189, June 1988.

[8] Yamagushi H., Kato H. (1983). - On application of nonlinear cavity flow theory to thick foil sections. I Mech E, $2^{\text {nd }}$ International conference on cavitation, Edinburg, pp. 164-174, September 1983.

[9] Reboud J.-L., Catelan F.-X., De Bernardi J., Stutz B. (1994). - Analyse et modélisation des poches de cavitation. CREMHyG, commande SEP/R\&T R24/N 847321, Mars 1994. 